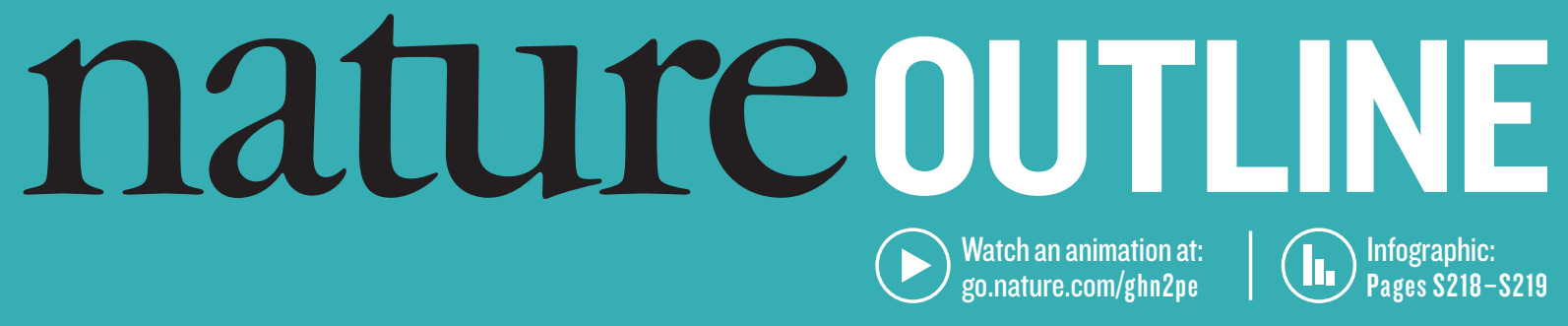

\title{
OVARIAN CANCER: BEYOND RESISTANCE
}

\section{Successfully treating the cancer requires overcoming the almost inevitable development of resistance to standard platinum-based therapy.}

\section{BY DAVID HOLMES}

varian cancer is the most common cause of gynaecological-cancer-associated death. Although the past 40 years have seen our knowledge of the disease advance, translating that improved understanding into a tangible clinical benefit has been a tortuous process. The last major breakthrough in treatment came 20 years ago, with the addition of a taxane (paclitaxel or docetaxel are commonly used to treat ovarian cancer) to one of the several variants of platinum-based chemotherapy that remain the mainstay of treatment. Since then, refinements to surgery and to the timing and delivery of chemotherapy have produced only slight improvements in outcomes. In the United States, for example, 5-year survival has inched up from about $40 \%$ in 1985 to a still parlous $45 \%$ today. By comparison, 5-year survival for breast cancer stands at $90 \%$.

Two factors account for much of the stubbornly high mortality and morbidity associated with ovarian cancer - late diagnosis and treatment resistance. There are currently no approved methods to screen for ovarian cancer, although promising preliminary results released earlier this year from the UK Collaborative Trial of Ovarian Cancer Screening may begin to change that. Around 60\% of women are diagnosed with late-stage disease that has already spread within the abdomen. As many as $80 \%$ of these women will respond well to initial treatment with platinum-based therapy, but almost all will experience multiple recurrences of disease, with ever shorter disease-free intervals. Ultimately, almost all of these women will die from the disease - and most will die from a disease that is resistant to platinum chemotherapy.

As with most solid malignancies, resistance to platinumbased treatment can be intrinsic or acquired, and is brought about through a bewildering array of mechanisms. From pumps that eject the drug from the cell to promoting the expression of genes that enable alternative growth pathways, cancer cells leave no stone unturned in their bid to survive and

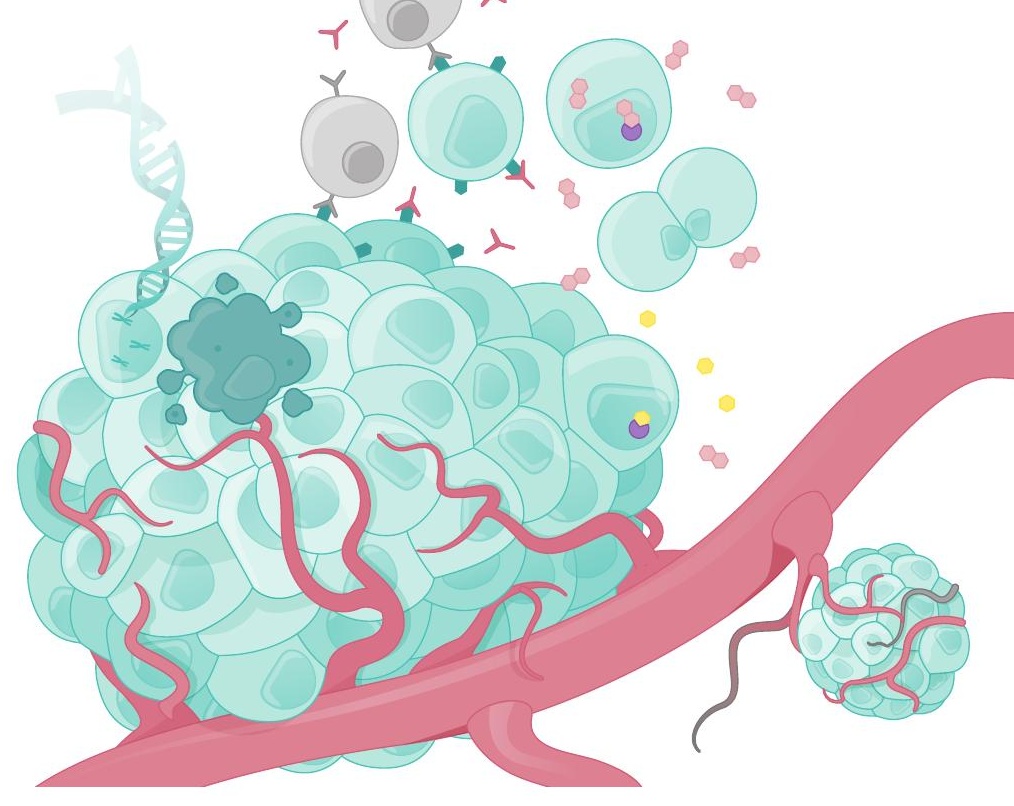

proliferate. Further complicating matters is the difficulty of knowing which mechanism or mechanisms are active in any particular person.

The good news is that there are a huge number of experimental therapies in development, and it is hoped that these can be added to platinum-based chemotherapy to help deliver a knockout blow, or at least prolong the intervals between treatment and improve patients' quality of life. Vaccines to activate the immune system against tumours, agents to interfere in DNA-repair pathways, and therapies that choke off the supply of blood to the tumour are all now in clinical trials for ovarian cancer. Because of the complexity of the disease and the mechanisms that underlie treatment resistance, it is unlikely that any one therapy will be a silver bullet. Nevertheless, there is a growing sense of optimism that researchers will be able to translate hard-won knowledge into improved outcomes for patients.

Nature is pleased to acknowledge the financial support of Pharma Mar, S.A. in producing this Outline. As always, Nature retains sole responsibility for all editorial content.

David Holmes is a science writer based in the United Kingdom.
Produced with support from:

\section{Pharma Mar}

Nature Outlines are sponsored supplements that aim to unpack scientific and technical concepts through infographics, illustrations and short animations. The boundaries of sponsor involvement are clearly delineated in the guidelines available at http://go.nature.com/ecx76b.

Feedback@nature.com. Copyright $\odot 2015$ Nature Publishing Group 